\title{
Reentrant transition of bosons in a quasiperiodic potential
}

\author{
A. Cetoli, E. Lundh \\ Department of Physics, Umeå University, SE-90187 Umeå, Sweden
}

PACS 64.70.Tg - Quantum phase transitions

PACS 03.75.Lm - Tunneling, Josephson effect, Bose-Einstein condensates in periodic potentials, solitons, vortices and topological excitations

\begin{abstract}
We investigate the behavior of a two dimensional array of Bose-Einstein condensate tubes described by means of a Bose-Hubbard Hamiltonian. Using a Wannier function expansion for the wavefunction in each tube, we compute the Bose-Hubbard parameters related to two different longitudinal potentials, periodic and quasiperiodic. We predict that - upon increasing the external potential strength along the direction of the tubes - the condensate can experience a reentrant transition between a Mott insulating phase and the superfluid one.
\end{abstract}

Introduction. - Since Greiner et al. first succeeded at storing a Bose-Einstein condensate in a two-dimensional array of narrow tubes using an optical lattice [1, experimental progress on low-dimensional Bose systems has been ' tremendous. To name but a few highlights, the Mott tran' sition was observed in three [2], one [3, and two dimen' sions [4, 5, and in 1D a Tonks-Girardeau gas has been realized 6, 7. Moreover, in 2D the Kosterlitz-Thouless transition was observed $[8,9$. The dimensional crossover between three, two, and one dimensions for bosons in an optical lattice was studied theoretically using TomonagaLuttinger liquid theory in Refs. [10,11 and using Monte Carlo simulations in Refs. [12,13]. These references studied 2D or 3D optical lattices in which atoms can tunnel easily along one Cartesian direction but not along the others. The system can then be described as an array of tubes of bosons, which may or may not be mutually phase coherent. As the tunneling between tubes is varied, such a system will undergo a transition from a 3D superfluid (3D SF) to a 2D Mott insulating phase (2D MI), which consists of a decoupled array of 1D tubes. Similarly, if the tunneling probability along all three Cartesian directions are made unequal, a 2D SF state can be realized, in which there is superfluidity within separate 2D layers but no coherence between them. These transitions are present only if the tubes have finite length.

The theoretical approaches used in Refs. 10 13] are able to describe phase fluctuations within the tube-like filaments of bosons, but they do not capture any nonlinear effects due to the possible variation in the width of these tubes. Gross-Pitaevskii theory describes such variations
14; however, it does so at the expense of not being able to account for phase fluctuations. Keeping these limitations in mind, we offer in this Letter a description that is complementary to those of Refs. 10 12, using GrossPitaevskii theory in order to understand how nonlinear effects may affect the phase transitions in this peculiar type of optical lattice.

Such effects become important if the potential barriers along the strongly coupled direction are so weak that each tube can be considered as a quasi-1D Bose-Einstein condensate and the number of bosons is large. It will be shown that these nonlinear effects can give rise to a reentrant Mott transition in the array of 1D tubes.

The dilute Bose gas in an external potential $V_{e x t}(\mathbf{r})$ is described by the second quantized Hamiltonian

$$
\begin{aligned}
\hat{H}\left[\hat{\psi}, \hat{\psi}^{\dagger}\right]=\int d \mathbf{r}\left[\frac{\hbar^{2}}{2 m}|\nabla \hat{\psi}(\mathbf{r})|^{2}\right. & +V_{\text {ext }}(\mathbf{r})|\hat{\psi}(\mathbf{r})|^{2}(1) \\
& \left.+\frac{g}{2}|\hat{\psi}(\mathbf{r})|^{4}\right]
\end{aligned}
$$

The physical setting consists of a two-dimensional optical lattice in the $x$ and $y$ plane with period $d$, creating a square array of tubes which develop along the $z$ direction. Moreover a weaker optical potential parallel to the tubes is added, so that in the present case the external potential is given by

$V_{\text {ext }}(x, y, z)=-V_{x} \cos \frac{2 \pi x}{d}-V_{y} \cos \frac{2 \pi y}{d}+V_{z}(z)$.

As in Ref. [15] the many-body field operator of the gas 
$\hat{\psi}(\mathbf{r})$ is rewritten as a sum of local operators

$$
\hat{\psi}(\mathbf{r})=\sum_{j_{x} j_{y} k} \phi_{j_{x} j_{y} k}(\mathbf{r}) \hat{b}_{j_{x} j_{y} k}
$$

where $\phi_{j_{x} j_{y} k}(\mathbf{r})=\phi_{k}\left(x+j_{x} d, y+j_{y} d, z\right)$ and each $\hat{b}_{j_{x} j_{y} k}$ acts on the $k^{t h}$ state of the tube in the position $\left(j_{x}, j_{y}\right)$. The $\phi_{k}$ are a complete set of wavefunctions. In the present setting we expect only the lowest energy state to be occupied, so that it is possible to drop the $k$ index from (3). Using the expression (3) the second quantized grand potential

$$
\hat{G}\left[\hat{\psi}, \hat{\psi}^{\dagger}\right]=\hat{H}\left[\hat{\psi}, \hat{\psi}^{\dagger}\right]-\mu_{\mathrm{G}} \hat{N}\left[\hat{\psi}, \hat{\psi}^{\dagger}\right]
$$

can be rewritten

$$
\begin{aligned}
\hat{G}= & -\sum_{j}\left[t_{x} \hat{b}_{j_{x}}^{\dagger} \hat{b}_{j_{x}+1}+t_{y} \hat{b}_{j_{y}}^{\dagger} \hat{b}_{j_{y}+1}+\text { h.c. }\right] \\
& +\frac{U}{2} \sum_{j} \hat{n}_{j}\left(\hat{n}_{j}-1\right)-\mu \sum_{j} \hat{n}_{j}
\end{aligned}
$$

where $j=\left(j_{x}, j_{y}\right), \hat{n}_{j}=\hat{b}_{j}^{\dagger} \hat{b}_{j}$, and the inter-tube tunneling matrix elements are

$$
\begin{aligned}
t_{\alpha}= & -\int d \mathbf{r}\left[\left(\nabla \phi_{j_{\alpha}}\right)^{*} \cdot \nabla \phi_{j_{\alpha}+1}\right. \\
& \left.+V_{\text {ext }}(x, y, z) \phi_{j_{\alpha}}^{*} \phi_{j_{\alpha}+1}\right],
\end{aligned}
$$

with $\alpha=\{x, y\}$. The in-tube interaction energy is

$$
\begin{gathered}
U=g \int d \mathbf{r}|\phi(\mathbf{r})|^{4}, \\
\mu=-\int d \mathbf{r}\left[\frac{\hbar^{2}}{2 m}|\nabla \phi(\mathbf{r})|^{2}+|\phi(\mathbf{r})|^{2} V_{\text {ext }}(\mathbf{r})\right]+\mu_{\mathrm{G}},
\end{gathered}
$$

where $\mu_{\mathrm{G}}$ is a constant that fixes the number $N_{\text {tot }}$ of particles in the whole system and $g=4 \pi \hbar^{2} a_{s} / \mathrm{m}$.

Finding the wavefunction $\phi(\mathrm{r})$ is a nontrivial task: We are ultimately concerned with approximating the full many-body ground state of the 3D system. In the absence of nonlinear on-site effects, the maximally localized Wannier functions can be used as a single-particle basis in which to expand the full many-body problem [15, 16. For the problem at hand, we are not only concerned with nonlinear effects from on-site repulsion, but also the elongated nature of the tubes. A rigorous recipe for how to choose the basis here cannot easily be formulated, but in principle the accuracy of the chosen basis could be assessed a posteriori by estimating how well the final solution approximates the 3D many-body ground state.

Nevertheless, we take guidance from the 1D singleparticle problem and apply the following ansatz to describe the wavefunction in each tube,

$$
\phi(\mathbf{r})=\sum_{i, j} a_{i j}(z) w_{i}(x) w_{j}(y),
$$
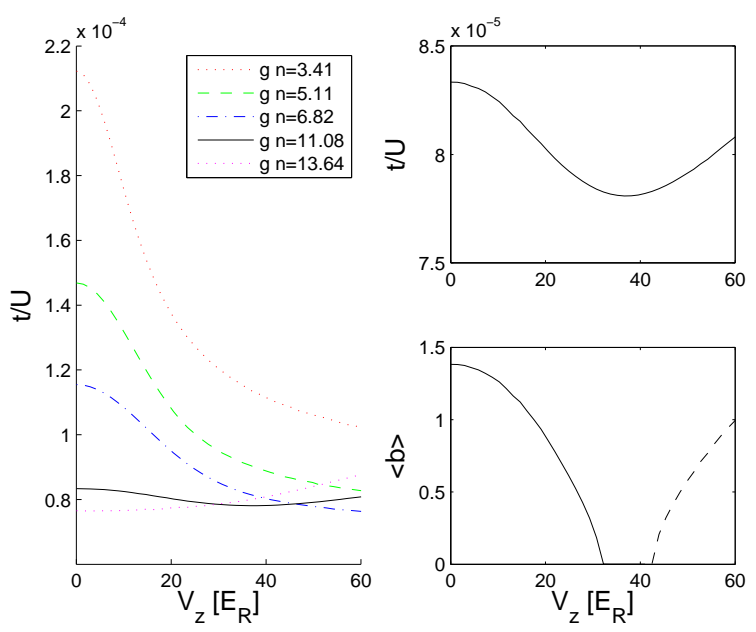

Fig. 1: Left panel: behavior of $t / U$ as a function of $V_{z}$ for some values of $g n$, using the the periodic potential (12) with $V=$ $77.35 E_{\mathrm{R}}$, as computed with the Wannier function approach. Upper right panel: behavior of $t / U$ as a function of $V_{z}$ for $g n=$ $11.08 E_{R}$. Lower right panel: tentative MF order parameter $\langle b\rangle$ against $V_{z}$ (see text) for the case $L=28(11.9 \mu \mathrm{m})$, and 28 particles for each tube.

where $\phi$ is normalized to unity

$$
\int d \mathbf{r} \phi(\mathbf{r})=1,
$$

and $w_{i}(x)$ and $w_{j}(y)$ are the Wannier functions for our lattice configuration along the $x$ and $y$ direction respectively. Since we expect the ground state in each tube to be symmetric, the sum employs only the even Wannier functions. The energy functional for the Bose-Einstein condensate in each tube is

$$
\begin{aligned}
H\left[\phi, \phi^{*}\right]=\int d \mathbf{r} & {\left[\frac{\hbar^{2}}{2 m}|\nabla \phi(\mathbf{r})|^{2}\right.} \\
& +V_{\text {ext }}(\mathbf{r})|\phi(\mathbf{r})|^{2} \\
& \left.+\frac{g n}{2}|\phi(\mathbf{r})|^{4}\right],
\end{aligned}
$$

with $n$ the mean number of particles in each tube. Inserting (9) in Eq. (11), an expression of the total energy as a function of the coefficients $a_{i j}(z)$ is obtained. The ground state has been found by minimizing this expression using the conjugate gradient method; in the chosen set of parameters it is enough to employ $0 \leq i, j \leq 4$, since the occupation number of higher Wannier states is negligible. Once the ground state is obtained, the coefficients $t$ and $U$ can be computed by means of (6) and (7).

Periodic potential. - Let us suppose that the potential along the tubes is periodic. For definiteness, let it have the same wavelength of the potentials in the $x$ and $y$ directions

$$
V_{z}(z)=V_{z} \cos \left(\frac{2 \pi}{d} z\right) .
$$


Focusing on the case in which $V=77.35 E_{\mathrm{R}}\left(E_{\mathrm{R}}\right.$ is the recoil energy $\hbar^{2} / m d$ ), in Fig. 1 (left panel) we plot the behavior of $t / U$ while varying $V_{z}$ from 0 to $60 E_{\mathrm{R}}$, for some values of the interaction strength. We see that for $g n=11.08 E_{R}$ the ratio $t / U$ decreases until $V_{z} \approx 39 E_{\mathrm{R}}$, then it begins to rise. It is known [17, 18, that the Mott insulating phase appears as a set of "lobes" in the plane $\mu / U-t / U$, each one corresponding to a precise number of particles $N_{\text {tube }}$ for each tube; outside of the 2D MI zone the system moves along lines of constant $N_{\text {tube }}$. The initial decrease and subsequent decrease of $t / U$ therefore means that for an appropriate length of the tubes the system can cross the 3D SF - 2D MI transition twice. In order to give an estimate of the critical potential strength, the Gutzwiller approximation is employed (see for example [18, 19]). The condensate order parameter is given by the expectation value of the destruction operator $\langle b\rangle$. Figure 1 (right lower panel) plots the behavior of this parameter for $L=28(11.9 \mu \mathrm{m})$ and 28 particles in each tube. The bounded interval in which the superfluid phase vanishes is a clear consequence of the nonlinear behavior of the system.

However, this example of a reentrant phenomenon does not survive a more accurate analysis. In fact, the parameters are such that when the system enters the 2D MI phase - thus isolating each tube from one another - the gas of bosons is in an insulating phase within the single tube. The assumption of coherence along the $z$ axis is not valid anymore, and a reentrance to a $3 \mathrm{D} \mathrm{SF}$ is questionable. Even changing the parameters, we were not able to find a case where the system remains superfluid along the tubes in the critical zone. This situation, added to the experimental difficulty to obtain the precise combination of parameters that realize the reentrant transition, makes the described phenomenon very unlikely to be observed in the case of a periodic potential along the tubes.

Quasiperiodic potential. - In order to avoid the Mott insulating phase within the tubes we employ instead a quasiperiodic potential

$$
V_{z}(z)=V_{z} \cos \left(\frac{2 \pi}{d} z\right)+V_{z} \cos \left(\frac{2 \pi}{d \lambda} z\right),
$$

where $\lambda=89 / 55$ is an approximation to the golden ratio. We have chosen to use the same lattice parameters as in the previous analysis, with the length of the tubes chosen to be $L=89 d(37.825 \mu \mathrm{m})$. Proceeding in this way we see a behavior similar to the one found for the periodic case: $t / U$ as a function of $V_{z}$ is a non-monotonic function for $g n=8.99 E_{R}$, as shown in Fig. 2. This situation gives rise to a reentrant transition when the number of particles in each tube is between 2500 and 2700. Figure2 (lower right panel) shows the mean field order parameter for $N_{\text {tube }}=$ 2600. According to the Gutzwiller approximation, in the shown parameter range there are a maximum of $\langle b\rangle^{2} \sim 100$ particles in the condensate.

In order to better understand the reason behind this
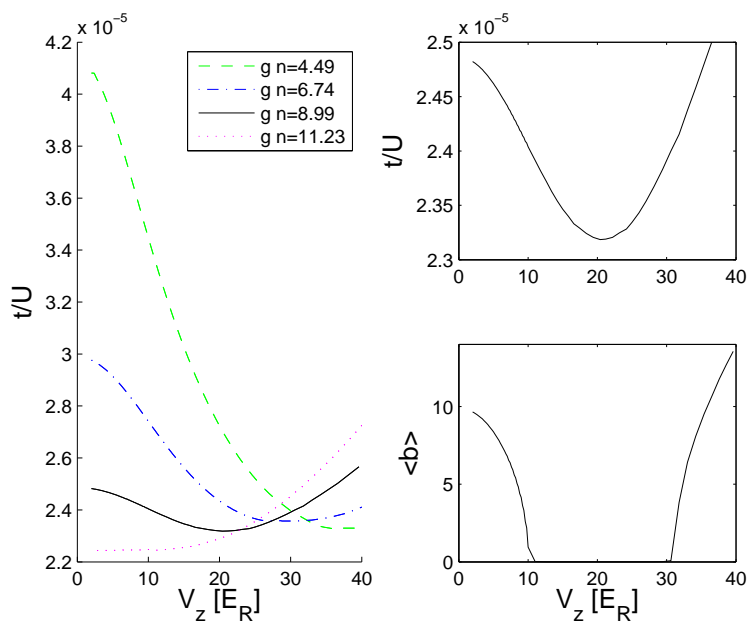

Fig. 2: Left panel: behavior of $t / U$ as a function of $V_{z}$ for the quasiperiodic case; the picture refers to some values of $g n$, while $V=100.0 E_{\mathrm{R}}$, as computed with the Wannier function approach. Upper right panel: behavior of $t / U$ as a function of $V_{z}$ for $g n=8.99 E_{R}$. Lower right panel: MF order parameter $\langle b\rangle$ against $V_{z}$ for the case $L=89 d(37.825 \mu \mathrm{m})$ and 2600 particles per tube.

phenomenon, the plot in Fig. 3 3 shows the behavior of $t$ and $U$ against $V_{z}$ separately for the case $g n=8.99 E_{R}$, and Fig. 目shows the density of the wavefunction $\int d y|\phi|^{2}$. Increasing the longitudinal quasiperiodic lattice we see that the peaks in the boson gas profile become narrower, thus raising the value of $U$ (17), while at the same time the wavefunction widens in the radial direction, increasing the tunneling rate $t$ (6). At first $U$ rises faster than $t$ but for $t \approx 25 E_{\mathrm{R}}$ this relation is reversed. The observed nonlinear effect is therefore the result of a competition between $U$ and $t$, i.e., between the increased density in a lattice minimum and the broadening of the tubes upon raising the longitudinal lattice strength.

In the presence of the quasiperiodic potential that we employ, the Mott insulator phase within a single tube is inhibited, but a new quantum phase comes along: the Bose glass. Recently a Bogoliubov analysis has been employed to trace the boundaries of the Bose glass phase 20121, and this method is expected to be accurate within the limit of the Bogoliubov approximation, i.e., weak interaction and high density $\left(g / n \ll \hbar^{2} / m[22]\right)$, as is the case for our current work. Using the same method we were able to determine that the single tube never leaves the superfluid phase during the reentrant phenomenon, thus making it possible for the system to experience twice the 3D SF2D MI transition while increasing the quasiperiodic lattice strength.

We thus conclude that our model is appropriate to predict the phase transition, and the consequent reentrant behavior.

Conclusions. - In this Letter we have studied the behavior of a 2D array of strongly elongated tubes of bosons 

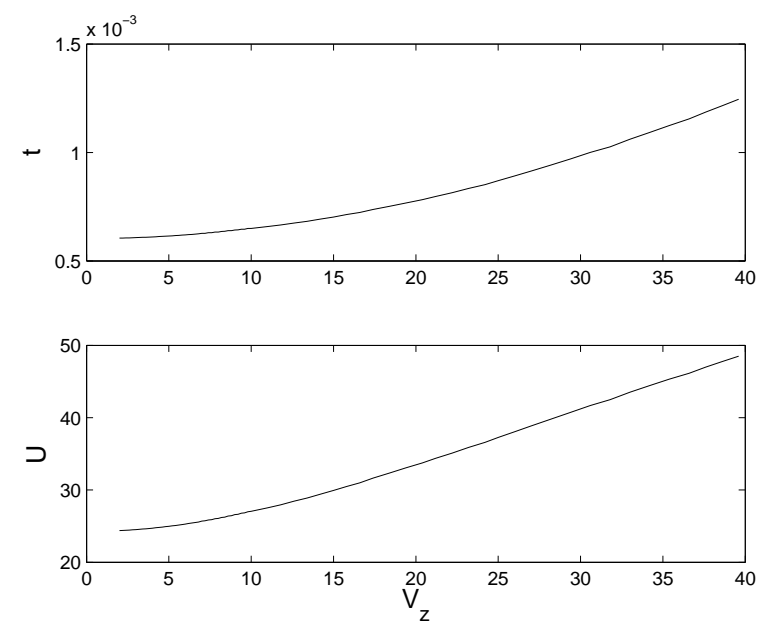

Fig. 3: Behavior of $t$ and $U$ as a function of $V_{z}$ for $g n=$ $8.99 E_{R}$, in the quasiperiodic case. While they both grow upon increasing $V_{z}$, the different rate at which they increase is responsible for the nonlinear behavior of $t / U$ (Fig. 2)

in an optical lattice, employing the Bose-Hubbard Hamiltonian in order to describe the 3D SF - 2D MI transition. Using a Wannier function ansatz and minimizing the Hamiltonian of the system we were able to compute the ground state in each tube, and link the parameter $t$ and $U$ of the Bose-Hubbard Hamiltonian to the physical parameters of the atom gas. Using this approach we were able to see a nonlinear behavior of the parameters, leading to the possibility of a reentrant transition between the 3D $\mathrm{SF}$ and the 2D MI phase.

We applied our method to two different potentials along the tubes: a periodic and a quasiperiodic modulation. While the non-monotonic behavior of $t / U$ is seen - for a specific choice of the parameters - in both cases, the periodic potential puts the tubes in a 1D MI phase, which would nullify the phenomenon we want to observe. In the quasiperiodic case, we predict that in an array of ${ }^{87} \mathrm{Rb}$ tubes $37.825 \mu \mathrm{m}$ long, with $d=425 \mathrm{~nm}$, $V_{x}=V_{y}=100 E_{\mathrm{R}}$, interaction strength $g n \sim 9 E_{R}$, and $N_{\text {tube }} \sim 2600$, the system goes through the 3D SF - 2D MI transition twice while increasing $V_{z}$. The system itself is found to be in the insulating phase for $V_{z}$ between $\sim 10 E_{R}$ and $\sim 30 E_{R}$. We stress that this is not a consequence of a localization phenomenon, but the quasiperiodic potential is only used to put the tubes in the right parameter regime.

Future prospects include investigating how the algebraic decay of phase coherence - which is not included in this study - affects the detailed values of the critical parameters. Moreover, a real experiment will employ an inhomogeneous trapping potential. This introduces the complication that not all the tubes would be equally long and the occupation number fluctuates. What is more likely to be observed in this situation is a dip in the phase coherence while varying $V_{z}$ in the range described above. Finally, we

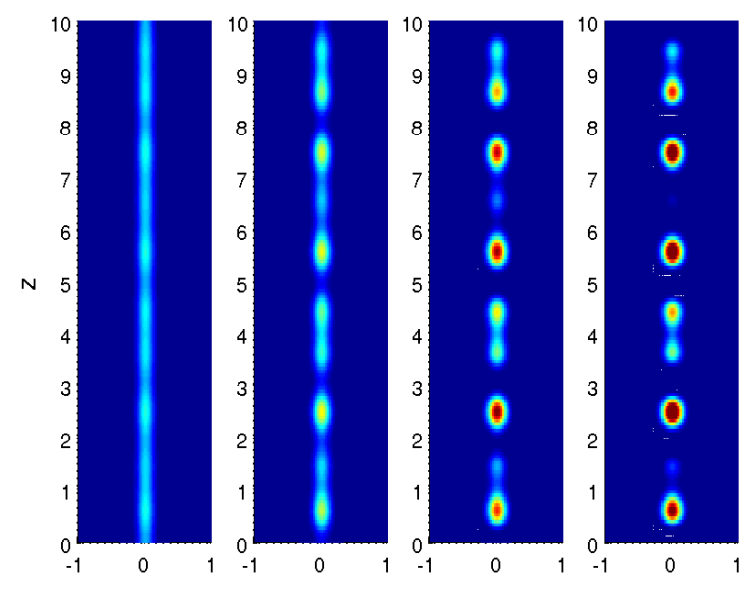

Fig. 4: Surface plot of the density profile $\int d y|\phi(x, y, z)|^{2}$ for - from left to right - $V_{z}=2,10,25$ and $40 E_{R}$, shown for $0<$ $z<10 d$. Increasing the longitudinal quasiperiodic lattice we see that the peaks in the boson gas profile become narrower, and at the same time the width of the wavefunction increases.

expect the combined quasiperiodic-plus-periodic potential under consideration to have a rich phase diagram if a wider parameter regime is considered; this phase diagram will include MI in different dimensions, Bose glass, and combinations thereof. The investigation of these phases will be the subject of a separate study.

\section{REFERENCES}

[1] Greiner M., Bloch I., Mandel O., Hänsch T. W., And Esslinger T., Phys. Rev. Lett., 87 (2001) 160405

[2] Greiner M., Mandel O., Esslinger T., Hänsch T. W., AND Bloch I., Nature, 415 (2002) 39

[3] Stöferle T., Moritz H., Schori C., Köhl M., AND Esslinger T., Phys. Rev. Lett., 92 (2004) 130403

[4] Köhl M., Moritz H., Stöferle T., Schori C., and Esslinger T., Journal of Low Temperature Physics, 138 (2005) 635

[5] Spielman I. B., Phillips W. D., and Porto J. V., Phys. Rev. Lett., 98 (2007) 080404

[6] Paredes B., Widera A., Murg V., Mandel O., Fölling S., Cirac I., Shlyapnikov G. V., Hänsch T. W., ANd Bloch I., Nature (London), 429 (2004) 277

[7] Kinoshita T., Wenger T., and Weiss D. S., Science, 305 (2004) 1125

[8] Schweikhard V., Tung S., and Cornell E. A., Phys. Rev. Lett., 99 (2007) 030401

[9] Hadzibabic Z., Krüger P., Cheneau M., Battelier B., and Dalibard J.B., Nature, 441 (2006) 1118

[10] Gangardt D. M., Pedri P., Santos L., and ShlyapNikov G. V., Phys. Rev. Lett., 96 (2006) 040403

[11] Ho A. F., Cazalilla M. A., and Giamarchi T., Phys. Rev. Lett., 92 (2004) 130405

[12] Bergkvist S., Rosengren A., Saers R., Lundh E., Rehn M., and Kastberg A., Phys. Rev. Lett., 99 (2007) 110401 
[13] Rehn M., Bergkvist S., Rosengren A., Saers R., Zelan M., Lundh E., And Kastberg A, Eur. Phys. J. D, 49 (2008) 223

[14] van Oosten D., van der Straten P., And Stoof H.T.C., Phys. Rev. A, 67 (2003) 033606

[15] Jaksch D., Bruder C., Cirac J. I., Gardiner C. W., ANd Zoller P., Phys. Rev. Lett, 81 (1998) 3108-3111

[16] Kohn W., Phys. Rev., 115 (1957) 4

[17] Fisher M. P. A., Weichman P. B., Grinstein G., And Fisher D. S., Phys. Rev. B, 40 (1) (1989) 546-570

[18] Sachdev S., Quantum Phase Transitions (Cambridge University Press) 1999, sect. 10

[19] Georges A., Proceedings of the International School of Physics "Enrico Fermi", 164 (2007) 477- 10

[20] Fontanesi L., Wouters M., and Savona V., Phys. Rev. Lett., 103 (2009) 030403

[21] Cetoli A., Lundh E., Submitted to PRA, ()

[22] Lieb E. H. And Liniger W., Phys. Rev., 130 (1963) 1605 